\title{
POINT DERIVATIONS IN CERTAIN SUP-NORM ALGEBRAS $\left.{ }^{(1}\right)$
}

\author{
BY \\ S. J. SIDNEY $\left({ }^{2}\right)$
}

1. Let $A$ be a closed point-separating subalgebra of $C(X)$ containing the constants, where $X$ is a compact Hausdorff space. $M_{A}$ will denote the space of multiplicative linear functionals $\varphi$ on $A$, and to each such $\varphi$ we associate its kernel $A_{\varphi}$. The $A_{\varphi}$ are precisely the maximal ideals of $A$.

Under certain hypotheses, it is known that analytic discs can be embedded in $M_{A}$. Wermer [W1] showed that if $A$ is a Dirichlet algebra on $X$, then each Gleason part of $A$ is either a single point or an analytic disc. Hoffman [H] then generalized Wermer's result to logmodular algebras. Finally Lumer [L] observed that the conclusion is really "local": if $\varphi$ has a unique representing measure on $X$, then the part for $A$ containing $\varphi$ consists either of $\varphi$ alone or of an analytic disc.

Our objective in this paper is to take the weakest of these possible hypotheses, namely Lumer's, and show that in a broader sense the analytic disc at $\varphi$, if there is one, really does account for all the analytic structure at $\varphi$. Specifically, we show that all the bounded derivations and higher "derivatives" of $A$ at $\varphi$ are just differentiations with respect to the analytic structure of the analytic disc.

2. All measures will be regular Borel measures on $X$; they will be nonnegative real-valued unless they are called complex, in which case they will be complexvalued.

Let $\varphi \in M_{A}$. Then there is a measure $\mu$ representing $\varphi$, i.e., $\varphi(f)=\int f d \mu$ for all $f \in A$. If there is only one such measure, we will say that $\varphi$ satisfies condition (U) (for unique). Lumer [L] has shown that condition (U) guarantees the validity of essentially all the logmodular theory of Hoffman's paper [H, $\$ \S 4-6]$ as applied to $\varphi$. We use this fact freely in the sequel. We shall require two additional facts based on Lumer's paper.

THEOREM 1. Suppose $\varphi \in M_{A}$ and $\mu$ is a measure representing $\varphi$. Then $\varphi$ satisfies (U) if and only if $\mu$ satisfies $\left(\mathrm{U}^{\prime}\right) \int u d \mu=\sup \{\operatorname{Re} \varphi(f): f \in A, \operatorname{Re}(f) \leqq u\}$ for all $u \in C_{R}(X)$.

Proof. This result is, even strongly generalized, quite familiar (see, for example,

Received by the editors December 20, 1966 and, in revised form, March 9, 1967.

(1) This work comprises a portion of the author's doctoral dissertation [S], written under the direction of Professors Andrew M. Gleason and Lynn H. Loomis and accepted by Harvard University in June 1966.

( $\left.{ }^{2}\right)$ Research partially supported by National Science Foundation Graduate Fellowship. 
[A]). Clearly $\left(U^{\prime}\right) \Rightarrow(U)$, since if $\nu$ were a second measure representing $\varphi$ we would have for all $u \in C_{R}(X)$

$$
\begin{aligned}
\int u d \mu & =\sup \{\operatorname{Re} \varphi(f): f \in A, \operatorname{Re}(f) \leqq u\} \\
& =\sup \left\{\int \operatorname{Re}(f) d v: f \in A, \operatorname{Re}(f) \leqq u\right\} \\
& \leqq \int u d v \leqq \inf \left\{\int \operatorname{Re}(f) d v: f \in A, \operatorname{Re}(f) \geqq u\right\} \\
& =\inf \{\operatorname{Re} \varphi(f): f \in A, \operatorname{Re}(f) \geqq u\}=\int u d \mu,
\end{aligned}
$$

hence $\int u d v=\int u d \mu$.

Conversely, suppose $\left(\mathrm{U}^{\prime}\right)$ is false and select $u \in C_{R}(X)$ such that $\int u d \mu>\alpha$ $=\sup \{\operatorname{Re} \varphi(f): f \in A, \operatorname{Re}(f) \leqq u\}$. Define a (real) linear functional $L$ on the (real) subspace of $C_{R}(X)$ spanned by $\operatorname{Re}(A)$ and $u$, setting $L(\operatorname{Re}(f)+r u)=\operatorname{Re} \varphi(f)+r \alpha$. It is easily seen that $L$ is a positive functional, hence has norm $L(1)=1$. Then one finds a measure $v$ representing $L$, i.e., $v$ represents $\varphi$ and $\int u d v=\alpha$. This last equality shows that $\nu \neq \mu$, hence (U) fails to hold.

Recall that the relation $\varphi \sim \varphi^{\prime} \Leftrightarrow\left\|\varphi-\varphi^{\prime}\right\|_{A^{*}}<2$ is an equivalence relation on points of $M_{A}$, and the equivalence classes are the (Gleason) parts for $A$ (see for example $[H, \S 7])$. We denote the part containing $\varphi$ by $P_{\varphi}$. The following fact is not actually necessary, but may make it easier for the reader to justify our use of some of Hoffman's results; combined with the aforementioned validity of \$4-6 of Hoffman's paper, it immediately guarantees in addition the validity of $\$ 7$ of Hoffman's paper for any $\varphi$ satisfying condition (U).

Theorem 2. Suppose $\varphi$ satisfies condition (U) and $\varphi^{\prime} \in P_{\varphi}$. Then $\varphi^{\prime}$ satisfies condition (U), and if $\mu$ and $\mu^{\prime}$ are the representing measures for $\varphi$ and $\varphi^{\prime}$ respectively, then $\mu$ and $\mu^{\prime}$ are mutually bounded absolutely continuous.

Proof. In the proof of Theorem 6 in [L], Lumer shows that if $\mu^{\prime}$ is any representing measure for $\varphi^{\prime}$ then $\mu^{\prime}$ is boundedly absolutely continuous with respect to $\mu$. Suppose now $\mu_{1}$ and $\mu_{2}$ are two representing measures for $\varphi^{\prime}$. Choose nonnegative real functions $h_{1}, h_{2} \in L^{\infty}(\mu)$ with $d \mu_{j}=h_{j} d \mu$. Then $g=h_{1}-h_{2} \in L^{1}(\mu)$ and $f \in A \Rightarrow \int f g d \mu=0$. Since $g$ is real-valued, $f \in A+\bar{A} \Rightarrow \int f g d \mu=0$. By Theorem 6.7 of $[\mathrm{H}], g=0$ a.e. $(\mu)$, so $\mu_{1}=\mu_{2}$.

3. A derivation of $A$ at $\varphi$ is a linear functional $D$ on $A$ satisfying the usual product rule for derivatives: $D(f g)=D(f) \cdot \varphi(g)+\varphi(f) \cdot D(g)$. The existence of a nonzero derivation at $\varphi$ is equivalent to $A_{\varphi} \neq A_{\varphi}^{2}$. [Note: If $I$ is an ideal in $A, I^{n}$ is the ideal generated by products $f_{1} \cdots f_{n}$ with $f_{i} \in I$ if $n \geqq 1$, and $I^{0}=A$; also, $I$ denotes the closure of $I$.] Similarly, the properness of the inclusion $A_{\varphi}^{3} \subset A_{\varphi}^{2}$ may be thought of as signifying a sort of second-derivative phenomenon, etc. In this same vein, the existence of a nonzero continuous derivation at $\varphi$ is equivalent to $A_{\varphi} \neq\left(A_{\varphi}^{2}\right)^{-}$, and so on. 
We can now state the main result of this paper.

THEOREM 3. Suppose $\varphi \in M_{A}$ satisfies condition (U). Then either $P_{\varphi}=\{\varphi\}$, in which case $A_{\varphi}=\left(A_{\varphi}^{2}\right)^{-}$and hence $A_{\varphi}=\left(A_{\varphi}^{n}\right)^{-}$for all $n \geqq 1$, or there is a homeomorphism $h$ of the open unit disc $D$ in the complex plane onto $P_{\varphi}$ (in the $A^{*}$ metric topology) such that $(D, h)$ is an analytic disc at $\varphi(i . e ., h(0)=\varphi$ and $\hat{f} \circ h$ is analytic for all $f \in A$, where $\hat{f}$ is the Gelfand transform of $f)$. In the latter case, $\left(A_{\varphi}^{n}\right)^{-} /\left(A_{\varphi}^{n+1}\right)^{-}$ is 1-dimensional and $\left(A_{\varphi}^{n}\right)^{-}$consists of those $f \in A$ for which $\hat{f} \circ h$ vanishes at 0 to order at least $n$, for each $n \geqq 0$.

The description of $P_{\varphi}$ as either a point or a disc is of course contained in the papers of Hoffman and of Lumer. Our contribution is the description of the ideals $\left(A_{\varphi}^{n}\right)^{-}$for $n \geqq 2$.

Theorem 3 is proved in the next section. In that section $\varphi$ will denote a multiplicative linear functional satisfying condition (U) and $\mu$ will denote its representing measure. Many of the arguments will look familiar, a point about which we shall make more comment later.

4. We begin with a mild variant of a standard result from the Dirichlet-logmodular theory [W2, Lemma 5].

LemMA 4. Suppose I is an ideal of $A, f \in L^{\infty}(\mu)$, and $f$ lies in the $L^{2}(\mu)$ closure of $I$. Then we can find a sequence $\left\{f_{n}\right\}$ in $I$ such that

$$
\left\|f_{n}\right\|=\sup \left\{\left|f_{n}(x)\right|: x \in X\right\} \leqq\|f\|_{\infty}
$$

and $f_{n} \rightarrow f$ a.e. $(\mu)$.

Proof. The proof is actually the same as Wermer's. One uses Theorem 1 instead of the Dirichlet property at a key point, and observes that if Wermer's $f_{n}$ are in $I$, so are his $h_{v}$. Glicksberg has also observed that this generalization holds [G, remark after Theorem 2.1].

Briefly, assume (as we may) that $\|f\|_{\infty}=1$ and let $\left\{k_{n}\right\}$ be a sequence in $I$ with $\left\|f-k_{n}\right\|_{2} \rightarrow 0$. Define $u_{n}=-\log ^{+}\left|k_{n}\right| \in C_{R}(X)$. One shows that $\int u_{n} d \mu \rightarrow 0$. Use Theorem 1 to pick $g_{n} \in A$ with $\operatorname{Re}\left(g_{n}\right) \leqq u_{n}, \operatorname{Im} \varphi\left(g_{n}\right)=0$, and $\operatorname{Re} \varphi\left(g_{n}\right)>\int u_{n} d \mu$ $-1 / n$. Then $\left\|\exp \left(g_{n}\right)\right\| \leqq 1$ and $\varphi\left(\exp \left(g_{n}\right)\right) \rightarrow 1$, from which one shows that $\exp \left(g_{n}\right) \rightarrow 1$ in $L^{2}(\mu)$. Setting $f_{n}=k_{n} \exp \left(g_{n}\right) \in I$, it follows that $f_{n} \rightarrow f$ in $L^{1}(\mu)$. Since $\left\|f_{n}\right\| \leqq 1$, a subsequence of $\left\{f_{n}\right\}$ satisfies the conclusion of the lemma.

Lemma 5. Suppose $L \in\left(A_{\varphi}^{n}\right)^{\perp}$ for a positive integer $n$, i.e., $L$ is a continuous linear functional on $A$ which annihilates $A_{\varphi}^{n}$. Let $\lambda$ be a complex measure representing $L$ and let $\lambda=\lambda_{a}+\lambda_{s}$ be its Lebesgue decomposition with respect to $\mu$. Then $\lambda_{a}$ represents $L$, i.e., $\lambda_{s}$ annihilates $A$.

Proof. We use induction on $n$. The case $n=1$ is the F. and M. Riesz theorem [H, Theorem 6.5]. Suppose $N>1$ and we know the lemma for $1 \leqq n<N$. Let $L \in\left(A_{\varphi}^{N}\right)^{\perp}$ have the complex representing measure $\lambda$. 
Let $g \in A_{\varphi}^{N-1}$ and define $L_{1} \in A_{\varphi}^{\perp}$ by $L_{1}(f)=L(f g)$. If $d \lambda_{1}=g d \lambda$ then $\lambda_{1}$ represents $L_{1}$, so the case $n=1$ tells us that $\left(\lambda_{1}\right)_{s}$ annihilates $A$, i.e., $f \in A \Rightarrow \int f g d \lambda_{s}=\int f d\left(\lambda_{1}\right)_{s}$ $=0$. Taking $f=1$ we obtain $\int g d \lambda_{s}=0$.

Thus $\lambda_{s}$ annihilates $A_{\varphi}^{N-1}$, so by our induction assumption $\lambda_{s}=\left(\lambda_{s}\right)_{s}$ annihilates A.

REMARK. A rather different and in some ways more satisfactory route to Lemma 5 is available. Ahern [A] has observed that, in considerably more generality than we need here, the F. and M. Riesz theorem can be made to follow from a lemma patterned after a theorem of Forelli [F, Theorem 1]. Glicksberg [G] has carried out this program in a form quite convenient for us: his proof of the $F$. and M. Riesz theorem [G, Theorem 1.1] from a Forelli-type lemma [G, Lemma 1.2] can be trivially modified to give our Lemma 5 for all $n$ simultaneously.

We have instead used the proof above for two reasons. First, Hoffman's paper $[\mathrm{H}]$ is our basic text, and our proof seems to be the quickest route from Hoffman's paper to Lemma 5. Second, this (admittedly trivial) proof is evidently applicable to a perhaps much larger class of situations: we have a projection $\lambda \rightarrow \lambda_{s}$ satisfying certain conditions (the conclusion of the F. and M. Riesz theorem) relative to an algebra $A$ and an ideal $I$, and we draw the same conclusion for the ideals $I^{n}$; it is possible to reformulate the entire affair in purely algebraic terms, and conclude that if a certain kind of projection "satisfies an F. and M. Riesz theorem" with respect to a suitable algebra $A$ and ideal $I$, then it also "satisfies an F. and $\mathrm{M}$. Riesz theorem" with respect to $A$ and $I^{n}$.

$H^{p}(\mu)$ denotes the closure of $A$ in $L^{p}(\mu), 1 \leqq p<\infty$, and $H^{\infty}(\mu)=H^{2}(\mu) \cap L^{\infty}(\mu)$. Lemma 4 and bounded convergence show that $H^{\infty}(\mu)$ consists of the a.e. $(\mu)$ pointwise limits of bounded sequences in $A$, hence is a Banach algebra when endowed with a.e. $(\mu)$ pointwise operations and the $L^{\infty}$ norm. Let $H_{\varphi}^{p}(\mu)$ $=\left\{f \in H^{p}(\mu): \int f d \mu=0\right\}, 1 \leqq p \leqq \infty$. In particular, if $1 \leqq p<\infty$, then $H_{\varphi}^{p}(\mu)$ is the $L^{p}$ closure of $A_{\varphi}$.

$\varphi^{\prime} \in P_{\varphi}$ has a unique representing measure $\mu^{\prime}$, and $\mu$ and $\mu^{\prime}$ are mutually boundedly absolutely continuous. Thus the spaces $L^{p}(\mu)$ and $L^{p}\left(\mu^{\prime}\right)$ are identical as function spaces, as are $H^{p}(\mu)$ and $H^{p}\left(\mu^{\prime}\right), 1 \leqq p \leqq \infty$, and the respective pairs of norms are equivalent.

For each $f \in L^{1}(\mu)$ we can therefore define a function $\hat{f}$ on $P_{\varphi}$ by $\hat{f}\left(\varphi^{\prime}\right)=\int f d \mu^{\prime}$. Clearly this agrees with the usual notion of $\hat{f} \mid P_{\varphi}$ if $f \in A$. Further, $f \rightarrow \hat{f}\left(\varphi^{\prime}\right)$ is a bounded linear functional on $L^{1}(\mu)$ (hence on $\left.L^{p}(\mu), 1 \leqq p \leqq \infty\right)$ for each $\varphi^{\prime} \in P_{\varphi}$.

LEMMA 6 (see [H, Theorem 5.1]). $f \rightarrow \hat{f}\left(\varphi^{\prime}\right)$ is multiplicative on $H^{2}(\mu)$ for each $\phi^{\prime} \in P_{\varphi}$, in the sense that if $f, g \in H^{2}(\mu)$ then $f g \in H^{1}(\mu)$ and $(f g)^{\wedge}\left(\varphi^{\prime}\right)=\hat{f}\left(\varphi^{\prime}\right) \cdot \hat{g}\left(\varphi^{\prime}\right)$. In particular, $f \rightarrow \hat{f}\left(\varphi^{\prime}\right)$ is multiplicative on $H^{\infty}(\mu)$.

LEMMA 7. If $L \in\left(A_{\varphi}^{n}\right)^{\perp}$ for a positive integer $n$, $L$ extends to $L_{1} \in L^{\infty}(\mu)^{*}$ in such a way that $\left\|L_{1}\right\|=\|L\|, L_{1}$ annihilates $\left(H_{\varphi}^{\infty}(\mu)\right)^{n}$, and $L_{1}$ is weakly continuous, i.e., if $\left\{f_{j}\right\}$ is a bounded sequence in $L^{\infty}(\mu)$ and $f_{j} \rightarrow g$ a.e. $(\mu)$ then $L_{1}\left(f_{j}\right) \rightarrow L_{1}(g)$. 
Proof. Select $\lambda$ a complex measure representing $L$ such that $\|\lambda\|=\|L\|$. Lemma 5 shows that $d \lambda=h d \mu$ for some $h \in L^{1}(\mu)$. Lemma 4 then can be used to see that $L_{1}(f)=\int f d \lambda$ will do.

THeOREM 8. Suppose $P_{\varphi} \neq\{\varphi\}$. Then there is a homeomorphism $h$ of the open unit disc $D$ onto $P_{\varphi}$ (in the $A^{*}$ metric topology) such that $(D, h)$ is an analytic disc at $\varphi$. If $h_{1}$ and $h_{2}$ are two such functions then $h_{1}^{-1} \circ h_{2}$ is an analytic homeomorphism of $D$ onto itself. Any such function $h$ satisfies:

(a) $\hat{f} \circ h$ is analytic for each $f \in H^{2}(\mu)$.

(b) If $L \in\left(A_{\varphi}^{n}\right)^{\perp}$ for a positive integer $n$ and $L_{1}$ is the extension of $L$ to $L^{\infty}(\mu)$ guaranteed by Lemma 7, then $L_{1} \mid H^{\infty}(\mu)$ has the form $L_{1}(f)=\sum_{k=0}^{n-1} a_{k}\left(d^{k} / d z^{k}\right)(\hat{f} \circ h)(0)$ for appropriate constants $a_{0}, \ldots, a_{n-1}$.

Proof. Theorems 7.6 and 7.4 of $[\mathrm{H}]$ imply the existence of a $Z \in H_{\varphi}^{\infty}(\mu)$ with the following properties:

(i) $|Z|=1$ a.e. $(\mu)$.

(ii) $\hat{Z}$ maps $P_{\varphi}$ (in the $A^{*}$ metric topology) homeomorphically onto $D$.

(iii) The function $f-\sum_{k=0}^{m}\left(\int \bar{Z}^{k} f d \mu\right) Z^{k}$ is in $Z^{m+1} H^{2}(\mu)$ whenever $f \in H^{2}(\mu)$ and $m$ is a nonnegative integer.

(iv) $\varphi^{\prime} \in P_{\varphi}, f \in H^{2}(\mu) \Rightarrow \hat{f}\left(\varphi^{\prime}\right)=\sum_{k=0}^{\infty}\left(\int \bar{Z}^{k} f d \mu\right) \hat{Z}\left(\varphi^{\prime}\right)^{k}$.

Then $h=\left(\hat{Z} \mid P_{\varphi}\right)^{-1}$ satisfies everything except (b), and we now verify (b). Define $a_{k}=L_{1}\left(Z^{k}\right) / k$ ! for $0 \leqq k \leqq n-1$. Then $a_{k}\left(d^{k} / d z^{k}\right)(\hat{f} \circ h)(0)=\left(\int \bar{Z}^{k} f d \mu\right) L_{1}\left(Z^{k}\right)$ for $f \in H^{2}(\mu)$. If $f \in H^{\infty}(\mu)$ then (i) and (iii) show that $f-\sum_{k=0}^{n-1}\left(\int \bar{Z}^{k} f d \mu\right) Z^{k}$ is in $Z^{n} H^{\infty}(\mu) \subset\left(H_{\varphi}^{\infty}(\mu)\right)^{n}$, so $L_{1}\left(f-\sum_{k=0}^{n-1}\left(\int \bar{Z}^{k} f d \mu\right) Z^{k}\right)=0$ and therefore

$$
L_{1}(f)=\sum_{k=0}^{n-1}\left(\int \bar{Z}^{k} f d \mu\right) L_{1}\left(Z^{k}\right)=\sum_{k=0}^{n-1} a_{k}\left(d^{k} / d z^{k}\right)(\hat{f} \circ h)(0)
$$

This proves (b).

Now let $h^{\prime}$ be a second function mapping $D$ homeomorphically and analytically onto $P_{\varphi}$. Then $h^{-1} \circ h^{\prime}$ is a homeomorphism of $D$ onto itself. Lemma 4 permits us to find a bounded sequence $\left\{f_{j}\right\}$ in $A$ such that $f_{j} \rightarrow Z$ a.e. $(\mu)$. Then $\hat{f}_{j} \mid P_{\varphi} \rightarrow \hat{Z}$ pointwise, so $\hat{f}_{j} \circ h^{\prime} \rightarrow \hat{Z} \circ h^{\prime}$ pointwise. Since $\hat{f}_{j} \circ h^{\prime}$ is analytic by hypothesis, so is $\hat{Z} \circ h^{\prime}=h^{-1} \circ h^{\prime}$. Thus $h^{-1} \circ h^{\prime}$ is a diffeomorphism. This also implies that (a) and (b) hold for $h^{\prime}$, completing the proof.

Proposition 9. Let $Z$ be as in Theorem $8, h=\left(\hat{Z} \mid P_{\varphi}\right)^{-1}, n$ a positive integer. Define $L$ on $A$ by $L(f)=\left(d^{n-1} / d z^{n-1}\right)(\hat{f} \circ h)(0)$. Then $L \in\left(A_{\varphi}^{n}\right)^{\perp}$, but $L \notin\left(A_{\varphi}^{n-1}\right)^{\perp}$.

Proof. Using the Cauchy integral representation for derivatives, $|L(f)|$ $\leqq(n-1) !\|f\|$, so $L \in A^{*} . f \in A_{\varphi} \Rightarrow f=f-\left(\int \bar{Z}^{0} f d \mu\right) Z^{0} \Rightarrow f \in Z H^{\infty}(\mu)$ by (iii), so $f \in A_{\varphi}^{n} \Rightarrow f=Z^{n} g$ where $g \in H^{\infty}(\mu)$. Therefore $(\hat{f} \circ h)(z)=z^{n} \cdot(\hat{g} \circ h)(z)$ where $\hat{g} \circ h$ is analytic on $D$, so $L(f)=0$. Therefore $L \in\left(A_{\varphi}^{n}\right)^{\perp}$.

On the other hand, Lemma 4 enables us to select a bounded sequence $\left\{f_{j}\right\}$ in $A_{\varphi}$ such that $f_{j} \rightarrow Z$ a.e. $(\mu)$. Then $\hat{f}_{j} \mid P_{\varphi} \rightarrow \hat{Z}$ pointwise, so $\hat{f}_{j} \circ h \rightarrow \hat{Z} \circ h$ pointwise. It follows that $L\left(f_{j}^{n-1}\right) \rightarrow(n-1)$ ! while $f_{j}^{n-1} \in A_{\varphi}^{n-1}$. Thus $L \notin\left(A_{\varphi}^{n-1}\right)^{\perp}$. 
Theorem 8 and Proposition 9 prove that portion of Theorem 3 dealing with the case $P_{\varphi} \neq\{\varphi\}$. The case $P_{\varphi}=\{\varphi\}$ will now be covered by showing that if $A_{\varphi} \neq\left(A_{\varphi}^{2}\right)^{-}$ then there is a nontrivial analytic disc at $\varphi$.

Let $V$ denote the closure in $L^{2}(\mu)$ of $A_{\varphi}^{2}$. Clearly $V \subset H_{\varphi}^{2}(\mu)$.

LeMma 10. $A_{\varphi} \neq\left(A_{\varphi}^{2}\right)^{-} \Rightarrow H_{\varphi}^{2}(\mu) \neq V$.

Proof. Select $f_{0}$ in $A_{\varphi}$ but not in $\left(A_{\varphi}^{2}\right)^{-}$. Then $f_{0} \in H_{\varphi}^{2}(\mu)$.

We can find $L \in\left(A_{\varphi}^{2}\right)^{\perp}$ such that $L\left(f_{0}\right) \neq 0$. Suppose $f_{0} \in V$. By Lemma 4 we can select a bounded sequence $\left\{f_{j}\right\}$ in $A_{\varphi}^{2}$ such that $f_{j} \rightarrow f_{0}$ a.e. $(\mu)$. On the one hand $L\left(f_{j}\right)=0$. On the other hand, Lemma 7 implies that $L$ is weakly continuous, hence $L\left(f_{j}\right) \rightarrow L\left(f_{0}\right) \neq 0$, a contradiction. Thus $f_{0} \notin V$.

THEOREM 11. If $A_{\varphi} \neq\left(A_{\varphi}^{2}\right)^{-}$there exists $G \in H_{\varphi}^{\infty}(\mu)$ satisfying

(i) $|G|=1$ a.e. $(\mu)$.

(ii) $G$ spans $H_{\varphi}^{2}(\mu) \cap V^{\perp}$.

(iii) $G H^{2}(\mu)=H_{\varphi}^{2}(\mu)$.

Proof. This will be a familar invariant subspace argument, and will really be the proof of the following more general theorem (see, e.g., [SW, Theorem 3.1]): If $M$ is a singly invariant closed subspace of $L^{2}(\mu)$ (i.e., the closed linear span of $A_{\varphi} M$ is a proper subspace of $\left.M\right)$ then $M=G H^{2}(\mu)$ where $|G|=1$ a.e. $(\mu)$.

By Lemma 10 we can select $G \in H_{\varphi}^{2}(\mu) \cap V^{\perp}$ such that $\|G\|_{2}=1$. We show that (i), (ii) and (iii) hold.

$$
f \in A_{\varphi} \Rightarrow G f \in V \Rightarrow G f \perp G \Rightarrow \int f|G|^{2} d \mu=0 .
$$

Thus $|G|^{2} d \mu$ represents $\varphi$, so uniqueness of $\mu$ gives (i).

If (ii) is false we can find orthonormal $G_{1}, G_{2} \in H_{\varphi}^{2}(\mu) \cap V^{\perp}$. Let $\left(a_{1}, a_{2}\right)$ be a pair of complex constants such that $\left|a_{1}\right|^{2}+\left|a_{2}\right|^{2}=1$. Then $a_{1} G_{1}+a_{2} G_{2} \in H_{\varphi}^{2}(\mu) \cap V^{\perp}$ and $\left\|a_{1} G_{1}+a_{2} G_{2}\right\|_{2}=1$, so again $\left|a_{1} G_{1}+a_{2} G_{2}\right|=1$ a.e. $(\mu)$. It is easily seen that this cannot hold simultaneously for all such pairs $\left(a_{1}, a_{2}\right)$. Therefore (ii) must be true.

In view of (i) and Lemma 6, $G H^{2}(\mu)$ is a closed subspace of $H_{\varphi}^{2}(\mu)$. Suppose $g \in H_{\varphi}^{2}(\mu) \cap\left(G H^{2}(\mu)\right)^{\perp}$. Then $f \in A \Rightarrow G f \in G H^{2}(\mu) \Rightarrow G f \perp g \Rightarrow \int \bar{f} \bar{G} g d \mu=0$. On the other hand, $f \in A_{\varphi} \Rightarrow f g \in V \Rightarrow f g \perp G \Rightarrow \int f \bar{G} g d \mu=0$. Thus $\bar{G} g d \mu$ annihilates $\bar{A}+A_{\varphi}$ $=A+\bar{A}$, so by Theorem 6.7 of $[\mathrm{H}] \bar{G} g=0$ a.e. $(\mu)$. Because of (i), $g=0$ a.e. $(\mu)$. Thus (iii) holds.

Lemma 12. Suppose $A_{\varphi} \neq\left(A_{\varphi}^{2}\right)^{-}$and $G$ is as in Theorem 11. Then whenever $f \in H^{2}(\mu)$ and $n$ is a nonnegative integer, we have $g_{n} \in G^{n+1} H^{2}(\mu)$ where

$$
g_{n}=f-\sum_{k=0}^{n}\left(\int \bar{G}^{k} f d \mu\right) G^{k}
$$

Proof. Induction on $n$.

With hypotheses as in Lemma 12 , for each $z \in D$ define a linear functional $\tilde{z}$ on 
$L^{2}(\mu)$ by $\tilde{z}(f)=\sum_{n=0}^{\infty}\left(\int \bar{G}^{n} f d \mu\right) z^{n} . \tilde{z}$ is bounded (with norm at most $\left.(1-|z|)^{-1}\right)$ and for each $f \in L^{2}(\mu)$ the function $z \rightarrow \tilde{z}(f)$ is analytic on $D$.

THEOREM 13. The map $z \rightarrow \tilde{z} \mid A$ is a nontrivial (in fact 1-1) analytic disc at $\varphi$. In particular, $A_{\varphi} \neq\left(A_{\varphi}^{2}\right)^{-} \Rightarrow P_{\varphi} \neq\{\varphi\}$.

Proof. Using Lemma 12 it is easy to see that $\tilde{z}$ is multiplicative on $H^{\infty}(\mu)$, hence on $A$; since also $\tilde{z}(1)=1, \tilde{z} \mid A \in M_{A}$. Clearly the map is "analytic", and Theorem 11 leads to $\tilde{0} \mid A=\varphi$. Thus $z \rightarrow \tilde{z} \mid A$ is an analytic disc at $\varphi$. Finally, select a sequence $\left\{f_{j}\right\} \subset A$ such that $f_{j} \rightarrow G$ in $L^{2}(\mu)$. Then for each $z \in D, \tilde{z}\left(f_{j}\right) \rightarrow \tilde{z}(G)=z$. Thus the map is $1-1$.

REMARK. Lemma 12 and Theorem 13 are essentially the argument used by Wermer in [W] and repeated by Hoffman in [H] to put a disc in $M_{A}$. Theorem 13 completes the proof of Theorem 3.

5. In this section we use our characterization of the ideal $\left(A_{\varphi}^{n}\right)^{-}$to see how certain behavior on $X$ of functions in $A$ can imply their belonging or at least being close to $\left(A_{\varphi}^{n}\right)^{-}$. Until further notice, we do not assume $\varphi$ satisfies condition (U).

If $\varphi \in M_{A}$, a Jensen measure for $\varphi$ on $X$ is a measure $\mu$ of a total mass 1 such that the "Jensen inequality" $\log |\varphi(f)| \leqq \int \log |f| d \mu$ holds for all $f \in A$. Such a $\mu$ is easily seen to be an Arens-Singer measure for $\varphi$ (i.e., $\log |\varphi(f)|=\int \log |f| d \mu$ for all invertible $f \in A$ ) and therefore a representing measure for $\varphi$ (since $\operatorname{Re}(A)$ $\subset \log \left|A^{-1}\right|$ ). Bishop has shown [B] that $\varphi$ always has a Jensen measure on $X$.

LEMMA 14. Suppose $\mu$ is a Jensen measure for $\varphi$ and $E$ is a Borel set in $X$ such that $\mu(E)>0$. Suppose $\left\{f_{j}\right\}$ is a sequence in $A$ and $g \in L^{1}(\mu)$ is such that $\left|f_{j}\right| \leqq g$ a.e. $(\mu)$, and suppose $f_{j} \rightarrow 0$ pointwise on $E$. Then $\varphi\left(f_{j}\right) \rightarrow 0$.

Proof. If $\varepsilon>0$ is given, select $\delta>0$ so small that $\left(\log (\varepsilon)-\int g d \mu\right) / \log (\delta)<\mu(E)$ and $\delta<1$. If $E_{j}=\left\{x \in E:\left|f_{j}(x)\right|<\delta\right\}$ we can find $J$ so large that $j \geqq J \Rightarrow \mu\left(E_{j}\right)$ $>\left(\log (\varepsilon)-\int g d \mu\right) / \log (\delta)$. Then

$$
\begin{aligned}
j \geqq J \Rightarrow \log \left|\varphi\left(f_{j}\right)\right| & \leqq \int \log \left|f_{j}\right| d \mu \\
& =\int_{E_{j}} \log \left|f_{j}\right| d \mu+\int_{x-E_{j}} \log \left|f_{j}\right| d \mu \\
& \leqq \mu\left(E_{j}\right) \log (\delta)+\int g d \mu<\log (\varepsilon),
\end{aligned}
$$

so $\left|\varphi\left(f_{j}\right)\right|<\varepsilon$.

LEMMA 15. Let $U$ and $V$ be open subsets of the complex plane with respective coordinates $u$ and $v$. Let $\tau: U \rightarrow V$ be analytic. Then for $1 \leqq k<\infty$ and $1 \leqq l \leqq k$, there exist polynomials $Q_{k, l}$ in $k$ variables $x_{1}, \ldots, x_{k}$ such that whenever $f$ is an analytic function on $V$, we have

$$
(f \circ \tau)^{(k)}(u)=\sum_{l=1}^{k} Q_{k, l}\left(\tau^{\prime}(u), \ldots, \tau^{(k)}(u)\right) f^{(l)}(\tau(u)), \quad 1 \leqq k<\infty .
$$

Proof. Induction on $k$. 
If $\left\{T_{\alpha}\right\}$ is a family of subspaces of $A$, a sequence $\left\{f_{j}\right\}$ in $A$ will be said to converge to $\left\{T_{\alpha}\right\}$, written $f_{j} \rightarrow\left\{T_{\alpha}\right\}$, if $\lim _{j}\left(\sup _{\alpha} \inf \left\{\left\|f_{j}-g\right\|: g \in T_{\alpha}\right\}\right)=0$. An easy application of the Hahn-Banach theorem shows that this is equivalent to the following: if $S$ is the closed unit ball in $A^{*}$, then $f_{j} \rightarrow 0$ uniformly on $\bigcup_{\alpha}\left(S \cap T_{\alpha}^{\perp}\right)$ where $f_{j}$ is interpreted as being in $A^{* *}$.

THEOREM 16. Suppose $\varphi$ satisfies condition (U) and $\mu$ is a representing measure for $\varphi$. Suppose $E$ is a Borel set in $X$ and $\mu(E)>0$. Suppose $\left\{f_{j}\right\}$ is a baunded sequence in $A$ such that $f_{j} \rightarrow 0$ pointwise on $E$. Then for each positive integer $n$,

$$
f_{j} \rightarrow\left\{A_{\varphi^{\prime}}^{n}: \varphi^{\prime} \in F\right\}
$$

where $F$ is any metrically compact subset of $P_{\varphi}$.

Proof. Let $\varphi^{\prime} \in P_{\varphi}$ have representing measure $\mu^{\prime}$. By Theorem $2 \varphi^{\prime}$ satisfies condition $(\mathrm{U})$ and since $\varphi^{\prime}$ has a Jensen measure on $X, \mu^{\prime}$ must be that Jensen measure. Lemma 14 then implies that $\varphi^{\prime}\left(f_{j}\right) \rightarrow 0$. Thus $\hat{f}_{j} \rightarrow 0$ pointwise on $P_{\varphi}$.

If $P_{\varphi}=\{\varphi\}$ then Theorem 3 says $\left(A_{\varphi}^{n}\right)^{-}=A_{\varphi}$ and we are done.

Assume $P_{\varphi} \neq\{\varphi\}$ and let $Z$ be as in Theorem $8, h=\left(\hat{Z} \mid P_{\varphi}\right)^{-1} \cdot\left\{\hat{f}_{j} \circ h\right\}$ is a bounded sequence of analytic functions on $D$ such that $\hat{f}_{j} \circ h \rightarrow 0$ pointwise, hence for every nonnegative integer $k,\left(\hat{f}_{j} \circ h\right)^{(k)} \rightarrow 0$ uniformly on any compact subset of $D$.

We must show that $f_{j} \rightarrow 0$ uniformly on $\bigcup\left\{S \cap\left(A_{\varphi^{\prime}}^{n}\right)^{\perp}: \varphi^{\prime} \in F\right\}$. We will accomplish this by finding a sequence of positive constants $\left\{c_{k}\right\}$ such that $L \in$ $S \cap\left(A_{\varphi^{\prime}}^{n}\right)^{\perp}$ for some $\varphi^{\prime} \in F$ implies $\left|L\left(f_{j}\right)\right| \leqq \sum_{k=0}^{n-1} c_{k}\left\|\left(f_{j} \circ h\right)^{(k)} \mid \hat{Z}(F)\right\|$. Since $\hat{Z}(F)$ is a compact subset of $D,\left(\hat{f}_{j} \circ h\right)^{(k)} \rightarrow 0$ uniformly on $\hat{Z}(F)$, and the theorem will be proved. We define the $c_{k}$ inductively by $c_{0}=1$ and $c_{k}=(1 / k !)+\sum_{s=0}^{k=1} c_{s} /(k-s)$ ! for $k>0$.

Suppose $\varphi^{\prime} \in F$. Let $Z^{\prime}$ be constructed for $\varphi^{\prime}$ as in Theorem 8 and set $h^{\prime}=$ $\left(\hat{Z}^{\prime} \mid P_{\varphi}\right)^{-1}$. Define an analytic function $\tau=\hat{Z} \circ h^{\prime}: D \rightarrow D$. ((a) in Theorem 8 shows that $\tau$ is indeed analytic.)

Let $L \in S \cap\left(A_{\varphi^{\prime}}^{n}\right)^{\perp}$ be given and let $L_{1}$ be the extension of $L$ guaranteed by Lemma 7. By Theorem $8 L_{1} \mid H^{\infty}(\mu)$ has the form $L_{1}(f)=\sum_{k=0}^{n-1} a_{k}\left(\hat{f} \circ h^{\prime}\right)^{(k)}(0)$. Lemma 15 then implies that $L_{1} \mid H^{\infty}(\mu)$ has the form $L_{1}(f)=\sum_{k=0}^{n-1} b_{k}(\hat{f} \circ h)^{(k)}\left(\hat{Z}\left(\varphi^{\prime}\right)\right)$. We will be done if $\left|b_{k}\right| \leqq c_{k}$ for all $k$. This we show by induction on $k$.

Observe that $\left|L_{1}\left(Z^{k}\right)\right| \leqq\left\|L_{1}\right\|=\|L\| \leqq 1$ for all $k \geqq 0$. Applied to the case $k=0$ this gives $\left|b_{0}\right| \leqq 1=c_{0}$.

Suppose $1 \leqq K \leqq n-1$ and $\left|b_{k}\right| \leqq c_{k}$ for $0 \leqq k<K$. Then

$$
\begin{aligned}
1 \geqq\left|L_{1}\left(Z^{K}\right)\right| & =\left|\sum_{s=0}^{n-1} b_{s}\left(\hat{Z}^{K} \circ h\right)^{(s)}\left(\hat{Z}\left(\varphi^{\prime}\right)\right)\right| \\
& =\left|\sum_{s=0}^{K} K ! b_{s}\left(\hat{Z}\left(\varphi^{\prime}\right)\right)^{K-s} /(K-s) !\right| \\
& =K !\left|b_{K}+\sum_{s=0}^{K-1} b_{s}\left(\hat{Z}\left(\varphi^{\prime}\right)\right)^{K-s} /(K-s) !\right|
\end{aligned}
$$


so that

$$
\left|b_{K}\right| \leqq \frac{1}{K !}+\left|\sum_{s=0}^{K-1} b_{s}\left(\hat{Z}\left(\varphi^{\prime}\right)\right)^{K-s} /(K-s) !\right| \leqq \frac{1}{K !}+\sum_{s=0}^{K-1} c_{s} /(K-s) !=c_{K} .
$$

Corollary 17. Let $\varphi, \mu$ and $E$ be as in Theorem 16. Suppose $f \in A$ and $f \mid E=0$. Then

$$
f \in \bigcap\left\{\left(A_{\varphi^{\prime}}^{n}\right)^{-}: \varphi^{\prime} \in P_{\varphi}, 1 \leqq n<\infty\right\}
$$

\section{REFERENCES}

[A] P. R. Ahern, On the generalized F. and M. Riesz theorem, Pacific J. Math. 15 (1965), 373-376.

[B] E. Bishop, Holomorphic completions, analytic continuation and the interpolation of seminorms, Ann. of Math. 78 (1963), 468-500.

[F] F. Forelli, Analytic measures, Pacific J. Math. 13 (1963), 571-578.

[G] I. Glicksberg, The abstract F. and M. Riesz theorem, J. Functional Analysis 1 (1967), 109-122.

[H] K. Hoffman, Analytic functions and logmodular Banach algebras, Acta Math. 108 (1962), 271-317.

[L] G. Lumer, Analytic functions and Dirichlet problem, Bull. Amer. Math. Soc. 70 (1964), 98-104.

[S] S. J. Sidney, Powers of maximal ideals in function algebras, Thesis, Harvard Univ., Cambridge, Mass., 1966.

[SW] T. P. Srinivasan and J. Wang, "Weak*-Dirichlet algebras," in Function algebras, edited by F. T. Birtel, pp. 216-249, Scott, Foresman and Company, Chicago, Ill., 1966.

[W1] J. Wermer, Dirichlet algebras, Duke Math. J. 27 (1960), 373-382.

[W2] — Seminar über Funktionen-Algebren, Lecture Notes in Mathematics, No. 1, Springer-Verlag, Berlin, 1964.

HARVARD UNIVERSITY,

Cambridge, Massachusetts

YALE UNIVERSITY,

New Haven, Connecticut 